\title{
Téoros
}

Revue de recherche en tourisme

\section{La prospective du tourisme méditerranéen (2000-2025)}

\section{Robert Lanquar}

Volume 6, numéro 3, décembre 1987

Le tourisme, phénomène mondial

URI : https://id.erudit.org/iderudit/1080601ar

DOI : https://doi.org/10.7202/1080601ar

Aller au sommaire du numéro

Éditeur(s)

Université du Québec à Montréal

ISSN

0712-8657 (imprimé)

1923-2705 (numérique)

Découvrir la revue

Citer cet article

Lanquar, R. (1987). La prospective du tourisme méditerranéen (2000-2025).

Téoros, 6(3), 15-18. https://doi.org/10.7202/1080601ar d'utilisation que vous pouvez consulter en ligne.

https://apropos.erudit.org/fr/usagers/politique-dutilisation/ 


\section{La prospective \\ du tourisme méditerranéen \\ (2000-2025)}

Robert Lanquar*

La Méditerranée est le premier bassin touristique du monde, comptant pour plus d'un tiers des arrivées du tourisme international et près de $10 \%$ des arrivées du tourisme national mondial. La croissance du tourisme y a été rapide et continue.

La poursuite de cette tendance est relle et doit servir au développement socio6conomique. Mais elle peut aggraver les phénomènes de saturation déjà visibles dans de nombreuses zones du littoral (dégradation des sites, murs de béton, eutrophisation maritime, disparition de la flore et de la faune terrestre, changements socio-culturels radicaux).

\section{Le Plan Bleu}

Le Plan Bleu est un exercice de prospective engagé par l'ensemble des pays méditerraneens dans le cadre du Plan d'action pour la Méditerrané, patronné par le Programme des Nations Unies pour l'Environnement. Le tourisme est une part importante de ce travail effectué pour micux comprendre les relations entre le développement et l'environnement dans les pays méditerrancens.

Cette exploration des évolutions possibles à long terme des rapports entre le développement et l'environnement en Méditerranée vise à mieux équiper les planificateurs et les décideurs des 18 pays du pourtour de la Méditerranée dans leurs choix et leurs activités portant sur le court et le moyen terme.

Comme les problemes de l'environnement ne deviennent alarmants que par leur accumulation dans un temps bien plus long que celui pris en compte habituellement dans le cal-

- M. Robert Lanquar ast directeur de I"IFDES - Burasu d'études de l'INFAC INFATH/CREAR/IDA. IEtudes, formations supérieures, développement international) et reside a Argenteuil en Franco. cul économique, il était indispensable de cerner de plus près la dynamique des interrelations entre les differents niveaux du couple économie-écologie, c’est-ä-dire du développement et de l'environnement, sur une plus longue période, de trois à cinq décennies.

C'est pourquoi les années 2000 et 2025 ont été déterminées comme horizons de scénarios pour décrire ces futurs possibles.

Les travaux du Plan Bleu se sont déroules en deux phases. La première phase a été un travail de reconnaissance limité à l'essentiel en raison de la tâche gigantesque de connaître tout ce qui a trait à la problématique environnement-développement dans l'ensemble du bassin méditerranéen.

Douze études ont êté effectuées autour des facteurs qui commandent ou déterminent le développement dans plusieurs secteurs dont le tourisme.

Plus de 120,000 donnes statistiques étaient rassemblées par l'équipe du Plan Bleu. De l'analyse de ces données, des tendances lourdes ont pu être identifiées. Elles encadrent les seénarios méditerranéens.

\section{La deuxième phase du Plan Bleu et le tourisme}

Il est en effet apparu nécessaire d'explorer d'autres modes de développement/environnement, à objectifs de niveaux de vie comparables pour analyser, sinon pour prévoir. au-delà des tendances lourdes ou des conflits déjà identifiés, des germes de mutations qui sont autant de facteurs qui imposent à toute action, un effort de réflexion prospective sur des alternatives (ou scénarios alternatifs).

Deux types de scénarios ont été présentés aux experts du Groupe de travail "Tourisme" qui ont élaborés les scénarios "tourisme" du Plan Bleu: des scénarios tendanciels ou référentiels contrastés et des scénarios alternatifs.

Les premiers ou scénarios " $\mathrm{T}$ " "ne marquent pas de fortes ruptures par rapport aux tendances observées jusqu’à maintenant avee, sur le plan international, des marges de manoeuvre modérées de la part des gouvernements de la Méditerranée. Ce sont des scénarios probables en raison des tendances suffissamment lourdes pour inflechir, dans. un sens ou dans un autre. les processus de la consommation touristique, soit au contraire la maintenir en état, malgré des contraintes d'ordre technique, financier...
Dans ces scénarios, le moteur économique est 1'expansion d'un marché international restant lié à une prépondérance économique nippo-américaine. En Méditerranće, les pays $s$ accomodent plus ou moins bien, au Nord comme au Sud, de cette prépondérance du Pacifique et les sociétés transnationales pesent lourd dans les évolutions.

Les scénarios " $\mathrm{A}$ ", alternatifs ou autocentrés, décrivent des évolutions qui s'écartent des tendances observées jusqu'à maintenant et une attitude plus volontariste de la part des gouvernements. Leur caractéristique est une plus grande affirmation des pays méditerranbens, permise par la formation d'une structure mondiale multipolaire.

Dans le scénario " A-I", les echanges méditerranéns seraient orientes Nord-Sud, l'Europe économico-politique aurait un rôle d'entraînement, mais naturellement nonhégémonique. C'est le scénario le plus favorable à la sauvegarde de l'environnement et à la lutte contre la pollution, avec une adoption possible des normes européennes que les pays du Nord aideraient financièrement a mettre en application; une telle aide s'ajoutant aux moyens nationaux.

Le scénario "A-2" se situe dans le mème contexte multipolaire. Ici, $\mathrm{I}^{+}$Europe a pourtant un rôle moins affirmé. Les pays riverains Est et Sud pourraient parvenir à une certaine unification, avec de nombreuses variantes possibles. On verrait l'effet bien connu d'une politique de développement autocentré, à savoir une intensification des echanges industriels et commerciaux entre partenaires. Ce scénario faciliterait l'équilibre rural-urbain et le développement du tourisme régional, national ainsi qu'entre pays limitrophes, certains pays etant plus favorisés que d'autres.

\section{Les perspectives à I'horizon 2000 et à I'horizon 2025}

L'utilisation de modèles de séries temporelles permet d'estimer pour l'année $2000_{z}$ le nombre de touristes internationaux dans la zone méditerranéenne à 173 millions.

Ceci équivaut à une croissance annuelle moyenne de $3 \%$, pratiquement au même niveau que le taux de croissance mondiale de la série temporelle, mais les hypothèses de croissance annuelle moyenne du nombre de touristes retenues varient selon les scénarios de 1,45 a $4,1 \%$. Il faut souligner que ces hypothèses sont relativement modestes si on les compare aux hypotheses que l'on 
trouve dans d'autres études aux horizons 90 et 95 et qui varient de 4 a $9 \%$ par an pour le tourisme international.

Quant à la demande touristique nationale, c'est-à-dire des nationaux des pays méditerranéens eux-mêmes qui atteignait en 1984, 105 millions d'arrivées, elle évoluerait selon le raisonnement que le tourisme national croitrait moins vite que le tourisme international, du fait que ceux qui en ont les moyens font déjà du tourisme.

Pour l'horizon 2025, on ne dispose que de résultats globaux. Par rapport à la situation existante en 1984, les coefficients multiplicateurs varient de 1,7 à 3,5 selon les scenarios. Les nombres de touristes sont à manier avec beaucoup de prudence, comme dans les autres scénarios à l'horizon 2025.

Les résultats de ces recherches sont présentées dans le tableau 1 à la page 16.

\section{Les perspectives d'évolution des comportements de voyages}

Une tentative a été faite de répartir de manière qualitative les touristes en Méditerranée, en essayant de cerner leurs activités préférentielles, leurs modes d'hébergement habituels ainsi que leur attitude en général face à l'organisation de leur temps de vacances.

L'idée directrice de cette tentative était de relier chaque type de touriste à des impacts spécifiques aussi bien sur l'environnement naturel que socio-culturel. Cette démarche est encore préliminaire, mais une enquête Delphi lancée auprès d'experts méditerranéens pour en tester la validité et vérifier la probabilité des hypothèses a montré que pour $3 / 4$ des experts, la répartition en 1985 était globalement vraisemblable.

Voici une estimation de cette répartition:

\section{Les perspectives d'évolution des rapports tourisme- environnement}

L'étude des relations entre le tourisme et l'environnement en Méditerranée a porté sur trois composantes:

- les prélèvements sur les ressources (ressources en eaux, emprise sur les sols);

- les pollutions et les déchets;

- les pressions physiques et socioculturelles.

Au total, les équipements d'hébergement touristique aurait déjà une emprise sur les sols des pays méditerranéens de plus de 2.168 $\mathrm{km}^{2}$, dont plus de $90 \%$ dans les trois pays les plus développés, 1"Espagne, la France et l'Italie. A l'horizon 2000, ce ne serait que 40 millions de lits touristiques qui existeraient sur les pays du pourtour méditerranéen, avec une hypothèse moyenne de croissance par rapport aux 33,1 millions de lits actuels.
TABLEAU 1

Le tourisme méditerranéen en 1984 et aux horizons 2000 et 2025 (nombre de touristes et de nuitées)

\begin{tabular}{lllllll}
\hline & \multicolumn{2}{l}{ Touristes (en millions) } & \multicolumn{3}{l}{ Nuitées (en millions) } \\
\hline & T.I & T.N & T.I+T.N & T.I & T.N. & T.I+T.N \\
\hline 1984 & 108 & 105 & 213 & 886 & 2.314 & 3.200 \\
\hline Scénarios 2000 & 181 & 127 & 308 & 1574 & 2955 & 4.529 \\
T1 & 162 & 106 & 268 & 1408 & 2.466 & 3.874 \\
T2 & 199 & 151 & 350 & 1731 & 3.513 & 5.244 \\
T3 & 207 & 168 & 375 & 1801 & 3.913 & 5.714 \\
A1 & 227 & 182 & 409 & 1970 & 4.238 & 6.208 \\
A2 & & & & & & \\
Scénarios 2025 & 311 & 169 & 480 & 2.553 & 3.727 & 6.280 \\
T1 & 265 & 113 & 378 & 2.172 & 2.485 & 4.657 \\
T2 & 343 & 230 & 573 & 2.814 & 5.073 & 7.887 \\
T3 & 356 & 306 & 662 & 2.917 & 6.729 & 9.646 \\
A1 & 409 & 348 & 757 & 3.352 & 7.661 & 11.013 \\
A2 & & & & & & \\
\hline
\end{tabular}

T.I.: Tourisme International T.N.: Tourisme National

Durée moyenne 1984: T.I.: 8,2 jours; T.N.: 22 jours

Durée moyenne 2000: T.1.: 8,7 jours; T.N.: 23,3 jours ( $+6 \%$ globalement)

Durée moyenne 2025: T.I.: 8,2 jours; T.N.: 22 jours

TABLEAU 2

Estimation de la répartition par types de touriste dans le bassin Méditerranéen en 1985 (d'après une enquête Delphi)

\begin{tabular}{lcc}
\hline & Pays méditer. du Nord & Pays méditer. du Sud \\
\hline - Aventuriers & $10 \%$ & $4 \%$ \\
- Villégiateurs & $40 \%$ & $80 \%$ \\
- Culturels & $25 \%$ & $8 \%$ \\
- Congressistes & $1 \%$ & $1 \%$ \\
- En-forme/santé & $7 \%$ & $2 \%$ \\
- Recréationnistes & $17 \%$ & $5 \%$ \\
\hline
\end{tabular}

TABLEAU 3

Le tourisme littoral* en 1984 et aux horizons 2000 et 2025 Nombre de touristes et nuitées

\begin{tabular}{lllllll}
\hline & \multicolumn{3}{l}{ Touristes (en millions) } & \multicolumn{3}{l}{ Nuitées (en millions) } \\
\hline & T.I & T.N & T.I+T.N & T.I & T.N. & T.I+T.N \\
\hline 1984 & 51 & 44,7 & 95,7 & 418,2 & 983,4 & $1.401,6$ \\
\hline Scénarios 2000 & & & & & & \\
T1 & 85,4 & 53,9 & 139,3 & 743 & 1.256 & 1.999 \\
T2 & 76,4 & 45 & 121,4 & 664 & 1.048 & 1.712 \\
T3 & 94 & 64,1 & 158,1 & 817 & 1,493 & 2.310 \\
A1 & 97,7 & 71,4 & 169,1 & 850 & 1.663 & 2.513 \\
A2 & 107 & 77,3 & 184,3 & 930 & 1.801 & 2.731 \\
\hline Scénarios 2025 & 147 & 72 & 219 & 1.205 & 1.584 & 2.789 \\
T1 & 125 & 48 & 173 & 1.025 & 1.056 & 2.081 \\
T2 & 162 & 98 & 260 & 1.328 & 2.156 & 3.484 \\
T3 & 168 & 130 & 298 & 1.377 & 2.860 & 4.237 \\
A1 & 193 & 148 & 341 & 1.582 & 3.256 & 4.838 \\
A2 & & & & & & \\
\hline
\end{tabular}

* Le tourisme littoral fut estimé à $47,2 \%$ du tourisme international méditerranéen et à $42,5 \%$ du tourisme national méditerranéen.

T.I: Tourisme International

T.N: Tourisme National

Durée moyenne 1984: T.I.: 8,2 jours; T.N.: 22 jours

Duré moyenne 2000: T.I. : 8,7 jours; T.N.: 23,3 jours (+ $6 \%$ globalement)

Durée moyenne 2025: T.I: 8,2 jours; T.N.: 22 jours 


\section{Emprise sur les sols: occupation du linéaire côtier}

Pour l'emprise sur les sols, l'hébergement touristique prendrait plus de $2.900 \mathrm{~km}^{2}$ en tenant compte de l'amélioration de la qualité, soit $3000 \mathrm{~km}$ de cotte totalement urbanisée sur $1 \mathrm{~km}$ de profondeur ou $6000 \mathrm{~km}$ de côte totalement urbanisée sur $500 \mathrm{~m}$ de profondeur.

\section{Consommation en eaux}

Il s'agit de consommation d'eau domestique. Les hypothèses reposent sur des pondérations. Un hôtel de luxe peut consommer plus de 600 litres d'eau par nuitée touristique alors qu'un campeur en utilise très peu. Au total, en 1984 on estime la consommation annuelle d'eau des touristes en Méditerrante à 569 millions de $\mathrm{m}^{3}$.

En 1'an 2000 et 2025, selon les scénarios, les coefficients multiplicateurs pourraient etre les suivants:

\begin{tabular}{lll}
\hline & 2000 & 2025 \\
\hline T1 & 1,7 & 2,6 \\
T2 & 1,5 & 2 \\
T3 & 2 & 3,2 \\
A1 & 2,1 & 3,9 \\
A2 & 2,3 & 4,4 \\
\hline
\end{tabular}

Le poids du tourisme dans la consommation d'eau n'est pas un problème insoluble, mais on doit y faire face longtemps à l'avance sur le plan technique et financier.

\section{Les déchets}

Quant aux déchets émis, les critères suivants ont été choisis:

- ordures ménagères par jour et par personne, $0,6 \mathrm{~kg}$ en zone rurale et $0,8 \mathrm{~kg}$ en zone urbaine;

- caux usées par jour et par personne, 150 litres.

En 1984, le volume des nuitées du tourisme méditerranéen aura été de 3200 millions de nuitées: cela veut dire que le volume des déchets des touristes s'est élevé à 2,2 millions de tonnes et celui des eaux usées ả 479 millions de $\mathrm{m}^{3}$.

En l'an 2000, si l'on prend l'hypothèse moyenne de croissance des nuiteses qui permet d'obtenir 4467 millions de nuitées pour l'ensemble des pays de la Méditerranée, le volume des déchets sera environ de 3,4 millions de tonnes (moyenne journalière 0,75 $\mathrm{kg}$ ) et celui des eaux usées de 670 millions de $\mathrm{m}^{3}$. Pour le seul littoral méditerranéen, les déchets touristiques seraient de 1,5 millions de tonnes et les eaux usees environ 300 millions de $\mathrm{m}^{3}$. Si les tendances continuaient arithmétiquement, en l'an 2025, tous ces chiffres tripleraient.

\section{Images des scénarios aux années 2000 et 2025}

Le scénario T1

Ce scénario se caractérise par la priorité don- née au tourisme international et à une emprise sur l'espace littoral à la limite de la saturation. C'est dans ce scénario que le nombre de villegiateurs, c'est-â-dire des touristes séjournant le long du littoral a la recherche de soleil, mer et plage, serait relativement le plus šlevé (au moins $40 \%$ en l'an 2000 et $42 \%$ en 2025).

Cela signifie que l'emprise sur l'espace par les résidences secondaires, les centres de villégiatures sera de plus en plus forte, en raison de la poursuite des tendances actuelles. L'offre d'hebergement croitra de plus de $20 \%$ dans ce sénario. Cela se traduirait par une exacerbation de la spéculation avec des conséquences désastreuses sur l'emprise des sols.

Certes, des solutions intermédiaires pourront être trouvées en étalant mieux les vacances, en bâtissant propres, surtout si l'on veut rentabiliser les hébergements qui seront de meilleure qualité pour les touristes internationaux qui d'ailleurs imposeront des normes et des standards de qualité. Petit à petit, le pourtour méditerranéen deviendra un "mur de béton" de résidences secondaires pour le tourisme national et d'appartements en location pour le tourisme international sur une frange de moins de 500 mètres.

C'est donc au niveau de la saturation des espaces que les difficultés les plus graves surviendront. Ce scénario peut-il alors être acceptable? Il comporte d'une part des injustices sociales, car le tourisme national croitra moins rapidement que la demande de vacances. Il implique un rejet du tourisme associatif et social qui devra se rentabiliser à tout prix et être amené à produire des prestations pour une clientêle plus aisée ne rentrant plus dans le cadre de ses objectifs premiers.

A la limite, pour éviter certaines pressions sociales, les pays les plus riches du Bassin méditerranten, pour se donner bonne conscience et esquisser quelques pas dans le cadre d'une politique sociale européenne, seront amenés à faire quelques efforts pour leurs nationaux et ceux des États voisins.

En définitive, le tourisme aurait moins d'impact sur l'économie qu'il ne l'aurait laissé prévoir $(5,8 \%$, moyenne méditerranéenne du tourisme dans le P.I.B.) et le nombre d'emplois ne croitrait que dans l'intervalle 8 a $11 \%$, la situation la plus dramatique, en raison des efforts de productivitée pour réduire la part de la main-d'oeuvre dans le coût des produits et services. Mais les salaires augmenteraient, ce qui explique la croissance de $5,8 \%$ du P.I.B.

A l'an 2025, ces tendances seraient tellement amplifiés qu'elles conduiraient à des étrangetés: le tourisme ne compterait que pour $5,6 \%$ du P.I.B.. Les arrivées du tourisme national et international, croissant au taux interannuel de $2,4 \%$, atteindraient $533 \mathrm{mil}-$ lions. Le littoral méditerranéen serait conpletement sature.
Des cités comme Benidorm, MalagaToremolinos, Athènes et sa banlieue touristique, Rimini deviendraient des mégapoles si denses qu'elles rencontreraient les mêmes problêtnes que les grandes capitales nationales ou régionales, avec des difficultés en matière de congestion des transports, d'approvisionnement d'eau potable et de trailtement des déchets et eaux usées. La seule note positive serait une part plus importante de la zone Est, si les problèmes politiques sont résolus à l'horizon 2025. En effet, la part de la zone constitube par l'Espagne, la France et l'Italie ne serait plus que de $71,9 \%$, celle du nord de l'Afrique croitrait jusqu'à $9 \%$ et le reste de la Méditerranée passerait entre les annees 2000 et 2025 de $15,5 \%$ à $19,1 \%$.

\section{Le scênario $\mathrm{T} 2$}

Le scénario " $\mathrm{T} 2$ " a été présenté comme un cas extrême: celui de la concurrence internationale la plus vive. Une déréglementation totale servirait les plus forts et handicaperait les faibles, d'autant que d'autres formes de réglementation pourraient se faire jour, biaisant les pratiques concurrentielles. Le tourisme national sera le grand perdant de ce scénario. Il sera paralysé, ne progressant plus, au moins jusqu"à l"année 2000 .

C'est aussi le scénario des AVENTURIERS, des SPORTIFS et du TOURISME DE SANTÉ. Les "Aventuriers" seront attires par les recoins encore "vierges" de la Mediterranée, certains "Sportifs" voudront "se défoncer" "durant leurs vacances pour être en forme physiquement dans leur milieu de travail et etre des professionnels plus performants et agressifs.

Le tourisme international, amplifie de 108 millions a 162 millions (une croissance de $50,4 \%$ ) serait en outre plus différencié quant aux styles et aux catégories socioéconomiques de vacanciers. Les intermédiaires des pays riches emetteurs de touristes tableraient sur des produits exotiques et très spécialisés et les pays développés seraient les principaux bénéficiaires du développement touristique.

C'est le scénario de l'agression publicitaire dans le monde du tourisme, le regne de la mode et de l'éphémère! Derrière une façade médiatisée belle et nette, les problèmes d'environnement s'aggraveront. La protection de la nature ferait plus l'objet de discours que de réalisations ênergiques. Des sites seront protégés comme des oasis pour touristes fortunés. Le reste, ce seront des équipements de moindre qualité dans des environnements moins beaux et moins propres, car les communautés locales n'auront pas les moyens de traiter les dechets et les eaux usees. Moins de touristes, mais plus de pollutions, voilà un autre aspect de ce scénario de la régression at de l'agression.

Ce scénario profitera pourtant à quelques pays du sud méditerranéen qui auront su se placer sur quelques créneaux porteurs. 
Le scénario $\mathrm{T} 3$, libéral et optimiste Ce scénario s'inscrit dans une vision à long terme pour améliorer la situation śconomique d'ensemble des pays de la Méditerranée. La croissance économique va permettre au tourisme national d'atteindre de bons résultats, mais ce ne seront pas les résultats d'une politique de justice sociale.

Dans cet ensemble, le tourisme national des pays de la Méditerranée compterait en l'an 2000 , pour $42,8 \%$ du marché touristique mediterranéen) et le tourisme international, $57,2 \%$ du total. Pour héberger, informer, occuper tout ce monde, il faudra bien que les opérateurs de tourisme innovent sans trop se fier aux interventions des Etats qui chercheront à garder une position libérale de "moins d'État" dans l'organisation des loisirs et des vacances des populations nationales.

Si I'on examine la répartition des flux touristiques à l'an 2000 et 2025 dans les trois grandes zones, le bloc de l'Espagne, la France et l'Italie, le Nord de l'Afrique et le reste de la Méditerranée, il semblerait que le bloc des trois pays les plus touristiques perdrait moins de sa part de marché que dans les scénarios $\mathrm{T} 1$ et $\mathrm{T} 2$.

Le nord de l'Afrique atteindrait facilement $8.5 \%$ du total des arrivées. Le reste de la Méditerranée stagnerait jusqu'en l'an 2000, puis en raison de conditions plus favorables augmenterait jusqu'à $18,5 \%$. Forte de sa croissance, 1'Europe aurait une politique de coopération tournée vers la Méditerranée comme pour se donner "bonne conscience". C'est-à-dire que les experts ont imaginé que l'aide serait "saupoudrée" dans les pays méditerranéens pour différents projets touristiques et de loisirs sans une coordination véritable et une bonne stratégie de développement.

Ensuite, la compétition commerciale sera moins sévêre, ce qui permettra aux pays méditerranéens de mieux ajuster leurs stratégies individuelles. Dans cet esprit, le développement de l'hinterland des littoraux et celui des îles pourrait conduire à une meilleure répartition du tourisme avec une plus grande influence sociale pour les pays moins développés et un meilleur équilibre entre tourisme, agriculture et industrie.

Cependant, il s'agit du scénario le plus grave pour l'environnement. Certes, des normes de pollution existeront (il ne faut pas craindre que les plages soient sales, elles seront nettoyées chaque matin et lessivées avec des bactéricides), mais elles ne seront pas assez contraignantes pour les aménageurs. D'ailleurs, si les scénarios T1 et T2 étaient ceux des hommes de promotion et de publicité, le scénario T3 sera celui des aménageurs et des hommes de marketing d'entreprise. Une note optimiste pourtant, les pressions sur le littoral seront telles que des seuils de saturation s'établiront vite et que la clientèle ira chercher une qualité de l'environnement dans les milieux ruraux de l'hinterland.
Le scếnario A1, scénario alternatif de co0pération ou scénario propre

Les scénarios alternatifs offrent de larges possibilités sur le plan théorique et pratique. Ce sont surtout des scénarios de solidarité volontariste: d'abord des solidarités locales entre les différents acteurs du développement touristique, ensuite des solidarités internationales entre les pays du Nord et ceux du Sud.

Dans le scénario "A.1" , les échanges méditerranéens seraient orientés Nord-Sud. l'Europe économico-politique aurait un certain rổle d'entraînement, mais naturellement non-hégémonique. Un cercle vertueux s'installerait sur le pourtour méditerranéen. Une meilleure croissance de l'Europe entrainerait une meilleure croissance du Sud et viceversa. Pourtant, ce rôle d'entraînement n'aurait pas des effets aussi positifs qu'on l'aurait pensé a priori.

En effet, si l'on examine les estimations élaborées, d'une part le tourisme méditerranéen croitra d'au moins $3,3 \%$ ( $3,2 \%$ dans le scénario "T. 3 ") par an d'ici l'an 2000 , puis de $2,0 \%$ de 2001 à 2025 ; d'autre part, la répartition régionale entre les trois pays du nordouest, le nord de l'Afrique et le reste de la Méditerranée donne des résultats sensiblement différents que dans les autres scénarios: c'est cette région composée de l'Espagne, la France et l'Italie, qui va plus bénéficier de la croissance du tourisme.

Le scénario ${ }^{4} \mathrm{~A} .1$." montre surtout la réussite des interventions des États industrialisés d'Europe dans le domaine social et le domaine écologique. C'est donc le scênario le plus favorable à l'aménagement du temps, à l'allongement des saisons touristiques et à l'étalement des vacances par des mesures coordonnées entre les États et les opérateurs de tourisme (différenciation des tarifs et des prix, obligation de ne pas prendre plus de 15 ou 20 jours de vacances en pleine saison, réorganisation des calendriers scolaires),

Le tourisme national en Méditerranée pourrait croitre autant, sinon plus que le tourisme international, ce qui donnerait une répartition $50 / 50 \%$ du tourisme national et du tourisme international en l'an 2000.

Les conditions économiques et politiques seront favorables à l'application par les pays industrialisés de l'Europe de normes très strictes en matière d'environnement qui s'imposeront aux aménageurs touristiques et aux stations de vacances du littoral pour éviter les saturations et pour gérer leur environnement.

Les espaces naturels seront largement conservés et on luttera contre la spéculation foncière par des mesures administratives appropriées. Peu de marinas seront encore ouvertes; les permis de construire pour les hebergements et les equipements touristiques ne seroni délivrés qu'au compte-goutte après de nombreux contrôles. L'Europe tentera d'imposer ses normes de protection de la nature aux pays moins développés pour faire de la Méditerranée, une mer propre, non polluée. La cooperation technique qu'elle dispensera largement, sera orientée dans cette direction. On n'aidera et ne prêtera qu'aux projets non polluants ou dépolluants. On évitera d'exporter ses touristes au Sud. On pourra même taxer par exemple les départs en vacances sur le littoral par des formules comme des taxes sur les eaux usees, les déchets, etc...

\section{Le scénario $\mathbf{A 2}$}

Le scénario "A.2" constitue une rupture par rapport aux tendances historiques et demande des estimations encore plus optimistes, basées sur les "meilleures années" de la croissance touristique, tout en restant dans des limites accessibles. Dans un tel scénario, le tourisme national aurait une croissance supérieure au tourisme international. La raison serait la très bonne santé économique des pays en développement qui récupéreraient leur retard de départs en vacances et d'utilisation du temps libre en activités de loisirs.

De 1984 à 2000 , le taux de croissance choisi par jugement et non pas selon un modele, serait de l'ordre de $4 \%$, comme aux plus beaux jours de la croissance touristique dans les années 50 et 60 . On atteindrait les 400 millions de touristes avec moins de $45 \%$ de touristes internationaux et plus de $55 \%$ de touristes nationaux. Puis à partir de l'année 2000 , la croissance s'essouflerait et ne serait plus que de $2 \%$ par an: vacanciers, touristes et excursionnistes seraient plus nombreux que les populations des pays méditerranéens!

Le réflexe de populations qui vont pour la première fois en vacances est de partír au bord de la mer. On peut se poser la question si la capacité de charge des littoraux méditerranéens n'atteindra pas des seuils au-delà desquels des dommages irréversibles seront faits à l'environnement: foréts incendiées, zones humides saccagées, nappes phréatiques asséchées... La concurrence pourrait alors être vive dans l'utilisation des ressources entre l'agriculture et le tourisme.

\section{Les lignes de l'alternance seraient donc:}

- la priorité donnée au secteur rural, done au tourisme rural dans le cadre d'une complémentarité avec l'agriculture;

- l'emphase portée sur le développement culturel de sa propre population, à travers les loisirs et les vacances;

- l'utilisation du tourisme comme moyen de formation et de promotion sociale. La formation servirait, plus que tout autre, de support à la coordination régionale et nord-sud. Elle serait non pas un leurre pour vendre ensuite des equipements, dans des cadres bilatéraux, mais une action de "bonne coopération", sans arrière pensée politique ou économique. Cette formation serait concrète, adaptée aux réalités et aux conditions économiques, sociales et religieuses de chaque pays, sinon de chaque zone territoriale développant son tourisme. 